\title{
Abandono del adulto mayor: una perspectiva sociodemográfica
}

\author{
Abandono do idoso: a sociodemographic perspective
}

Abandono do idoso: uma perspectiva sociodemográfica

\author{
Sadith Raquel Cotrado Huamán \\ sara_cotrado@hotmail.com \\ https://orcid.org/0000-0001-9785-3672
}

Universidad Peruana Unión, Lima, Perú

\author{
Guido Angelo Huapaya Flores \\ angelo.huapaya@upeu.edu.pe \\ https://orcid.org/0000-0002-1237-2886
}

Universidad Peruana Unión, Lima, Perú
RESUMEN

El objetivo del estudio fue determinar la relación de los factores sociodemográficos con la percepción de abandono del adulto mayor en la ciudad de Lima en el periodo del 2016. La metodología utilizada fue: cuantitativo; descriptivo, exploratorio, asociativo, correlacional, multivariado con diseño: no experimental, Transversal; en una población de adulto mayor 1463200 en Lima y en Chosica 40,000 . Los resultados describen que los factores sociodemográficos más de uno guardan relación significativa: género $(0,01)$ y actividad económica $(0,03)$. Concluyendo que el sólo hecho de pasar a la etapa de adulto mayor; sin menoscabar el nivel socioeconómico o el género o el estado civil que sea o la religión que práctica o con quién viva o la cantidad de hijos que tenga igual puede estar expuesto a sentirse abandonado; percibir afecto o abandono; o sentir que tiene soporte o no, o percibir tener cuidado por parte su familia o sentirse abandonado.

Palabras clave: Envejecimiento; adulto mayor; ancianidad; percepción afectiva; percepción de soporte; percepción de cuidado

ABSTRACT

RESUMO

The objective of the study was to determine the relationship of sociodemographic factors with the perception of abandonment of the elderly in the city of Lima in the period of 2016. The methodology used was: quantitative; descriptive, exploratory, associative, correlational, multivariate with design: non-experimental, Crosssectional; in an elderly population 1 463200 in Lima and 40,000 in Chosica. The results describe that more than one sociodemographic factors are significantly related: gender (0.01) and economic activity (0.03). Concluding that the mere fact of passing to the stage of older adult; Without undermining the socioeconomic level or the gender or the marital status or the religion that he practices or with whom he lives or the number of children he has the same, he may be exposed to feeling abandoned; perceive affection or abandonment; or feel that you have support or not, or perceive that your family is caring or feeling abandoned.

Key words:

Aging; Elderly; old age; affective perception; perception of support; perception of care
O objetivo do estudo foi verificar a relação dos fatores sociodemográficos com a percepção de abandono dos idosos na cidade de Lima no período de 2016. A metodologia utilizada foi: quantitativa; descritivo, exploratório, associativo, correlacional, multivariado com delineamento: não experimental, transversal; em uma população idosa 1 463200 em Lima e 40.000 em Chosica. Os resultados descrevem que mais de um fator sociodemográfico estão significativamente relacionados: sexo $(0,01)$ e atividade econômica $(0,03)$. Concluindo que o simples fato de passar ao estágio de adulto mais velho; Sem prejudicar o nível socioeconômico, o gênero, o estado civil ou a religião que pratica ou com quem vive ou o número de filhos que tem, pode estar exposto a sentir-se abandonado; perceber afeto ou abandono; ou sentir que tem apoio ou não, ou perceber que sua família está se preocupando ou se sentindo abandonada.

Palavras-chave: Envelhecimento; Idoso; velhice; percepção afetiva; percepção de suporte; percepção de cuidado 


\section{INTRODUCCIÓN}

La Organización Mundial de la Salud (OMS, 2016) revela una problemática de escala mundial: cerca de 1 de cada 10 personas mayores ha sufrido malos tratos en el último mes. Las tasas de maltrato pueden ser mayores entre los ancianos residentes en instituciones que en los que están en la comunidad. El maltrato de las personas mayores puede conllevar graves lesiones físicas y consecuencias psicológicas prolongadas. La Organización Mundial de la Salud resalta: se prevé un aumento del problema por el envejecimiento de la población en muchos países. La población mundial de mayores de 60 años se duplicará con creces, de 900 millones en 2015 a unos 2000 millones en 2050.

LA OMS describe la magnitud del problema refiriéndose: como un problema importante de salud pública. Aunque hay poca información sobre el alcance del maltrato en la población de edad avanzada, especialmente en los países en desarrollo, se calcula que 1 de cada 10 personas mayores ha sufrido malos tratos en el último mes. Probablemente la cifra esté subestimada, puesto que solo se notifica 1 de cada 24 casos de maltrato a personas mayores, en parte porque los afectados suelen tener miedo de informar a sus familiares y amigos o a las autoridades. En consecuencia, es probable que todas las tasas de prevalencia estén subestimadas.

En Latinoamérica describe el CEPAL (2002) que la región debe enfrentar el reto que plantea el enorme aumento de la proporción de población de 60 años y más, que pasa de $8 \%$ en el año 2000 a $14.1 \%$ en el año 2025 y a $22.6 \%$ en el año 2050. En cada país, los cambios futuros estarán relacionados con la situación actual y con la evolución futura de los indicadores demográficos. En la actualidad, el envejecimiento está más avanzado en Uruguay,
Argentina, Cuba y Chile y en varios países del Caribe (Trinidad y Tabago y Barbados). En estos países, más del $10 \%$ de la población es mayor de 60 años. En el otro extremo se sitúan países menos avanzados en su transición demográfica, como Guatemala, Bolivia, Paraguay, Honduras, Haití y otros, donde menos de un $6.5 \%$ de la población sobrepasa los 60 años. Hay un grupo de países en situación intermedia, cuya transición demográfica ya está bastante avanzada.

Por otro lado es importante resaltar que un rasgo distintivo del proceso de envejecimiento es el predominio femenino. Debido a la mayor sobrevida de las mujeres, se produce un aumento muy pronunciado de su proporción en las edades más avanzadas. Los índices de masculinidad a nivel regional presentan para el año 2000 un promedio de 86 hombres por cada 100 mujeres de 60 a 74 años, cifra que desciende a 70 hombres por cada cien mujeres en el grupo de 75 años y más.

Una realidad que expresa la situación crítica en la región es lo que sucede en México, Ramírez (2014) resalta que las personas en situación de abandono son altamente propensas a padecer maltrato, por ello, y conforme a una encuesta realizada por el Colegio de México en 2009 en el Distrito Federal, "se detectó que los adultos mayores son maltratados principalmente por las y los hijos, con un $37 \%$, seguidos de la pareja, con $11 \%$; las y los nietos, $10 \%$, mientras que personas ajenas representan 17.4\%". En los hogares se desvalorizan las capacidades, potenciales y autoestima de las personas de edad al ser ignorados, desatendidos y discriminados (entendiendo por discriminación la práctica cotidiana consistente en tratar de manera desfavorable, excluir o despreciar sin causa alguna a determinada persona o grupo, que a veces percibimos pero que en algún momento hemos generado o recibido). 
Mendoza (2012) en un reportaje para el diario la Republica titulado "La mitad de ancianos en provincias no son visitados por sus hijos" hace referencia que según estudio. Una encuesta reveló que adultos mayores de Arequipa se sienten abandonados por sus hijos y otros familiares. Esto es un tipo de maltrato, afirman especialistas. Expresa un caso de "Isabel vive en Islay, hace poco enviudó y se siente más sola que nunca. Sus hijos la visitan una vez cada dos meses. Esta sensación la entiende María, quien pasa sus días en un asilo ubicado en la calle Santa Rosa. La mujer también viene de provincia y ya no se acuerda cuándo fue la última vez que vio a su hijo único"

Igualmente, RPP (2012) en un reportaje describe la triste realidad del anciano en Lima: “Las grietas en la piel, lejos de ser símbolo de experiencia y sabiduría se han convertido en signos de maltrato e indiferencia. En nuestra capital habita el cincuenta por ciento de la totalidad de adultos mayores del país, muchos de ellos son dejados a su suerte". Introduce una interrogante: ¿Cuál es el motivo del maltrato y abandono a las personas de la tercera edad?, resalta lo que dijo la Dra. Carmen Gonzales, las personas descargan su resentimiento en las poblaciones más desvalidas. El reportaje concluye con la siguiente preocupación "Hoy en día se puede hablar de personas de la cuarta edad, aquellos mayores a 80 años. Este grupo se incrementa cada vez más al aumentar la esperanza de vida en el Perú, y son ellos quienes tienen aún más necesidades, tales como seguridad económica, salud e infraestructura. Lima Metropolitana cuenta con 6 residencias para adultos mayores, en las cuales se atiende a 600 personas, sin embargo la ayuda no es suficiente. Según Rocío Cutipé, jefa de la División de personas Adultas Mayores de la Municipalidad de Lima, es necesario fortalecer la atención a este grupo humano invirtiendo en infraestructura y personal capacitado".
Por lo tanto, la presente investigación se planteó como objetivo general: Determinar la relación de los factores sociodemográficos con la percepción de abandono del adulto mayor.

\section{Concepciones referenciales}

Si hablamos específicamente del abandono del adulto mayor podemos decir que el abandono es el descuido de los familiares hacia la persona mayor. Si hablamos judicialmente el abandono es un delito que consiste en poner en peligro la vida o la salud de una persona incapaz de valerse por sí misma. Este delito puede consumarse por no entregar el dinero necesario para su supervivencia o por la omisión de los cuidados personales.

\section{Tipos de abandono}

1. Maltrato físico: este tipo de abuso es muy frecuente en los asilos donde las personas que los cuidan se cansan de ellos y los golpean, también se da en las propias familias donde no tienen ningún respeto por ellos.

2. El maltrato psicológico o emocional: este tipo de maltrato seda en todo lugar ya sea en la calle casa o asilo pues la sociedad misma enseña o fomenta a esto.

3. El abuso patrimonial o maltrato económico; este tipo de maltrato hoy en día es común en la clase alta o media donde los hijos tratan de aprovecharse de las posesiones de sus padres, limitándoles muchas veces de su bienestar propio. Estas personas manejan a los ancianos como títeres para obtener beneficios económicos para ellos. 


\section{Teorías del adulto mayor}

A pesar de los numerosos esfuerzos que se han hecho por determinar las manifestaciones fenotípicas propias del envejecimiento, no se ha logrado llegar a un consenso entre las partes involucradas en la investigación. Además, el tema se ha abordado desde diversas perspectivas, que incluyen análisis teóricos con influencia religiosa o filosófica y también estudios científicos que se centran en los cambios fisiológicos que este proceso abarca.

Muchas de las teorías que se conocen hoy sobre este tema tienen sus raíces en preconceptos antiguos que han perdurado a través del tiempo y han sido adoptados incluso en la actualidad. A continuación, analizaremos algunos planteamientos que se han considerado más representativos y las modificaciones que han sufrido pese a algunas inconsistencias que estos presentaban.

\section{Teoría de Gestalt}

La teoría de Gestalt es en sí un conjunto de leyes que hablan de la percepción del ser humano. Si enfocamos cada ley al aspecto psicológico podemos decir:

Primeramente, el principio general de figura y fondo afirma que cuando se tiene una imagen sin fondo nuestro cerebro tratara de descifrar que es lo que es en realidad y llega a confundir a la mente. Esto provoca confusión y estrés en la persona. Esto mismo ocurre cuando una persona tiene una situación incierta en su vida y no sabe porque pasa lo que pasa; la persona tiene mucho estrés.

En segundo lugar, la ley general de la buena forma establece que el cerebro prefiere formas simétricas, continuas y acabadas que sean armoniosas. Muchas personas rechazan las cosas que no son así y también a persona que no son correctas o que no tienen una personalidad definida o firme. Nuestro cerebro siempre intentara definir lo inconcluso.

Como tercer aspecto cabe mencionar la ley del cierre o de la completud indica que cuando nos dan una figura inconclusa nuestro cerebro lo descifrará rápidamente puesto que tenemos la capacidad de hacerlo; en las situaciones cotidianas nosotros percibimos algunos aspectos de un problema y podemos sacar conclusiones y buscar una solución.

En cuarto lugar, la ley del contraste habla sobre el contraste de colores para poder percibir las cosas con claridad, llevándolo a las situaciones cotidianas podemos decir que es la capacidad de comparar situaciones para que así analicemos nuestra condición.

En quinto lugar, la ley de la proximidad establece que los elementos tienden a agruparse con los que están más próximos, las personas también lo hacemos por eso es algo lógico que estemos más apegadas a las personas con las que convivimos, es lo que pasa con los ancianos ellos sienten un gran apego por sus familiares y tratan de excusarlos para sentirse bien.

Finalmente, la ley de la similaridad afirma que los elementos que son iguales son agrupados en uno solo. Esto es lo que pasa hoy en día, pensamos que todos son iguales y pensamos que las personas que tienen características similares son iguales y tendemos a calificarlas igual que tras y a estereotiparizarlas (Gurevicz y Toro, 2014).

\section{Teorías psicológicas}

Esta teoría enfoca principalmente los siguientes temas: (a) Facultades cognitivas, (b) rasgos de la personalidad y (c) afrontamiento de la problemática del envejecimiento. Estos se explicarán detenidamente a continuación:

Si hablamos de las facultades cognitivas abarcamos un tema bastante amplio pues implica hablar de la capacidad de pensar, razonar, actuar, 
percibir, reaccionar, decidir y más. Y como hemos venido diciendo todos los ancianos sufren un deterioro cognitivo, pero unos más que otros. Esto puede ser influenciado por distintos aspectos como el nivel educativo, el entorno social en que viven y las actividades que realizo durante su vida. Todos estos aspectos influyen ampliamente en la personalidad de cada anciano, algunos pueden llegar a olvidar el idioma puesto que hay muchos quechua hablantes que tienen como segundo idioma el español y conforme pasan los años olvidan el español y les perjudica en sus relaciones personales.

También ocasiona el aislamiento de la persona porque nadie lo entendería. Por lo que puede tornarse introvertido y llegar a la depresión. Muchos ancianos al ver los cambios de la vejez no aceptan su situación y se frustran tratando de ocultar estos cambios ya sea con cirugías, maquillaje y otros. Estos con el paso de los años logran aceptar su vejez puesto que ya no pueden ocultarlo. Hay otras personas que aceptan la vejez y logran vivir felices y se desarrollan logrando una vejez óptima y saludable (Cobo, 2010).

\section{Teorías sociales}

Esta teoría explica que la sociedad ve al anciano como una carga que no genera ingresos, sino que genera gastos. Nuestra sociedad, hoy en día está interesada en personas jóvenes que puedan generar ingresos no en los ancianos los cuales bajan su rendimiento laboral por los mismos achaques de la vida. Los adultos mayores adquieren un estilo de vida sosegado, menos ajetreado en lo social, basado principalmente en ampararse bajo el cariño y el afecto de sus seres queridos; quienes son su principal soporte.

Sin embargo, muchos estudiosos dicen lo contrario, que al adquirir un estilo de vida súper activo en esta etapa de la vida lograra tener una vejez plena. En cambio, otros logran unir estas dos posiciones logrando una estabilidad entre ambas. Considerando que los ancianos tienden a autoexcluirse de las actividades físicas debido a que se sienten incapaces de realizarlas o consideran que las actividades que le piden hacer son vergonzosas (Cobo, 2010).

\section{Teoría del desarrollo de Erick Erickson}

En la fase del envejecimiento, Erickson veía un conflicto entre la integridad y la desesperación, en otras palabras, la aceptación de la vida individual en contra posición a los sentimientos de desesperación y depresión, donde se experimenta el miedo a la muerte y a las enfermedades existentes en esta última etapa de la vida. Además, esta se caracteriza por un distanciamiento social, un sentido de inutilidad biológica debido a que el cuerpo ya no responde como antes, junto a un sinnúmero de enfermedades somáticas propias de la edad, provocando la aparición de preocupaciones relativas a la muerte.

Los familiares, amigos y personas contemporáneas a su época mueren, y ello contribuye al nacimiento de un sentimiento de desesperanza. Esto promueve que algunos adultos mayores empiecen a preocuparse por su pasado, y en un proceso de reminiscencia, evalúen que han hecho con su vida a través de los años. Debido a la etapa natural del ciclo de vida que están pasando, el autor menciona que psicológicamente el adulto mayor se siente deprimido y preocupado no solo por su pasado (hechos que realizaron y que marcaron su vida), sino además por su futuro (la muerte y el sufrimiento que pasarán si se llegase a contraer alguna enfermedad) (Bordignon, 2005).

\section{Teoría de la Actividad o del Apego}

Es una teoría contraria a la teoría de la desvinculación o del desapego. Este modelo 
sociológico afirma que solo el individuo activo puede ser feliz y satisfecho. La persona ha de ser productiva, útil en el servicio material en el contexto donde se encuentre; de lo contrario, la desgracia, el descontento, la sensación de inutilidad se centrará en él. En esta se destaca la importancia de permitir que una persona mayor pueda realizar las mismas actividades que una persona joven, pues la diferencia de edad no debe ser un obstáculo, y evitar de esa manera que la persona mayor se sienta inútil e inservible ya que no realiza las mismas actividades que solía hacer en su juventud (Merchán y Cifuentes, 2013).

\section{MÉTODO}

El estudio es de enfoque cuantitativo de tipo no experimental de diseño descriptivo porque describe el nivel de abandono del adulto mayor en los hogares geriátricos y adultos mayores independientes, sin manipular deliberadamente la variable. Diseño asociativo, correlacional, comparativo porque se buscó hallar la relación que tiene las variables en estudio además de verificar las diferencias en el nivel de abandono en ambas muestras. De corte transversal porque el instrumento utilizado en la investigación se aplicó en un tiempo único.

La población está representada por adultos mayores a partir de los 60 años en adelante que gozan de buena salud mental y residen en hogares geriátricos y también adultos mayores que viven independientemente y adultos mayores que viven bajo el cuidado de sus hijos.

\section{Muestra}

En la definición del tamaño de la muestra se empleó el muestreo no aleatorio, no probabilístico; es decir; la selección y tamaño de la muestra estuvo a decisión del investigador.

Tabla 1. Distribución de la muestra de internos en los Hogares Geriátricos.

\begin{tabular}{lc}
\hline Hogar geriátrico & Cantidad \\
\hline Hogar Geriátrico Señor de los Milagros & 16 \\
Hogar Geriátrico Canevaro & 24 \\
Total & $\mathbf{4 0}$ \\
\hline
\end{tabular}

Tabla 2. Distribución de la muestra de Adultos Mayores Independientes.

\begin{tabular}{lc}
\hline DISTRITO & CANTIDAD \\
\hline Lima Metropolitana & 48 \\
Chosica & 32 \\
Total & $\mathbf{8 0}$ \\
\hline
\end{tabular}

\section{Técnicas de recolección de datos}

La técnica empleada para recolectar datos fue una encuesta que evalúa la percepción de abandono en el adulto mayor. El instrumento fue extraído de un estudio "Percepción del Adulto
Mayor Hospitalizado en Cuanto al Abandono por sus Familiares" con enfoque en la teoría de Gestalt; los autores fueron Amparo Zúñiga Ramos, Patricia Eugenia Pasquel Barrios y Aurora Zamora Mendoza; adaptado por el Mg. Guido Ángelo Huapaya Flores, 
teniendo en cuenta los indicadores y la situación de la población en estudio. Además, se elaboraron dos tipos de encuestas etiquetadas como A y B. La de tipo A fue utilizada para el estudio de los residentes de Hogares Geriátricos, mientras que la de tipo $B$ fue aplicada en adultos mayores que viven de manera independiente o dentro del vínculo familiar. Sin embargo, debemos destacar que las variaciones entre los dos tipos de instrumentos son mínimas y las preguntas corresponden a las mismas dimensiones según el orden establecido. $\mathrm{A}$ fin de determinar el nivel de Abandono del Adulto Mayor, ya sea que residan en Hogares Geriátricos o independientemente.

El instrumento fue nombrado "Cuestionario del Nivel de Abandono del Adulto Mayor" y está conformado por 28 preguntas y afirmaciones con ítems de respuesta cerrada (dicotómicos). El instrumento consta de instrucciones, información general y cuestionario. El tiempo calculado para rellenar la encuesta es de 20 minutos, considerando algunos contratiempos o problemas mencionados anteriormente.

El cuestionario está dividido en tres dimensiones, cada dimensión tiene indicadores diferentes. La dimensión de percepción afectiva consta de un solo indicador (Emocional) y 3 ítems, la dimensión de percepción de soporte consta de 3 indicadores (Familiar, Social y Económico) y 6 ítems y la dimensión de percepción de cuidado consta de 3 indicadores (Interés Familiar, Apoyo Sanitario y Personal de Apoyo) y 5 ítems. La distribución de estos fue de manera salteada para disminuir la posibilidad de sesgo.

\section{RESULTADOS}

La mayoría de los encuestados tipo A tienen asistencia médica de control en $87,5 \%$. Mientras que la mayoría del tipo B tienen esta asistencia en $51,3 \%$. La mayoría de los encuestados tipo A tienen atención personalizada permanente en $97,5 \%$. Mientras que la mayoría del tipo B tienen esta atención en $48,8 \%$. La mayoría de los encuestados tipo A tienen percepción de maltrato en $85 \%$. Mientras que solo la minoría del tipo B tienen esta atención en $73,8 \%$.

Tabla 3. Frecuencia del abandono del adulto mayor tipo A y tipo B.

\begin{tabular}{|c|c|c|c|c|c|c|c|c|c|c|c|c|}
\hline \multirow[b]{3}{*}{ Afectivo emocional } & \multicolumn{6}{|c|}{ Tipo A } & \multicolumn{6}{|c|}{ Tipo B } \\
\hline & \multicolumn{2}{|c|}{ SI } & \multicolumn{2}{|c|}{ NO } & \multicolumn{2}{|c|}{ TOTAL } & \multicolumn{2}{|c|}{ SI } & \multicolumn{2}{|c|}{ NO } & \multicolumn{2}{|c|}{ TOTAL } \\
\hline & $\mathbf{n}$ & $\%$ & $\mathbf{n}$ & $\%$ & $\mathbf{n}$ & $\%$ & $\mathbf{n}$ & $\%$ & $\mathbf{n}$ & $\%$ & $\mathbf{n}$ & $\%$ \\
\hline $\begin{array}{l}\text { Enfermedad Crónica } \\
\text { Degenerativa }\end{array}$ & 27 & 67.5 & 13 & 32.5 & 40 & 100 & 34 & 42.5 & 46 & 57.5 & 80 & 100 \\
\hline Percepción de Respeto Familiar & 26 & 65 & 14 & 35 & 40 & 100 & 68 & 85 & 12 & 15 & 80 & 100 \\
\hline Satisfacción Afectiva y Emocional & 25 & 62.5 & 15 & 37.5 & 40 & 100 & 61 & 76.3 & 19 & 23.8 & 80 & 100 \\
\hline \multicolumn{13}{|l|}{ Soporte familiar } \\
\hline Percepción de Abandono & 26 & 65 & 14 & 35 & 40 & 100 & 54 & 67.5 & 26 & 32.5 & 80 & 100 \\
\hline Responsabilidad Familiar & 37 & 92.5 & 3 & 7.5 & 40 & 100 & 58 & 72.5 & 22 & 27.5 & 80 & 100 \\
\hline $\begin{array}{l}\text { Participación en Actividades } \\
\text { Recreativas Familiares }\end{array}$ & 8 & 20 & 32 & 80 & 40 & 100 & 51 & 63.8 & 29 & 36.3 & 80 & 100 \\
\hline
\end{tabular}




\begin{tabular}{|c|c|c|c|c|c|c|c|c|c|c|c|c|}
\hline & \multicolumn{6}{|c|}{ Tipo A } & \multicolumn{6}{|c|}{ Tipo B } \\
\hline & \multicolumn{2}{|c|}{ SI } & \multicolumn{2}{|c|}{ NO } & \multicolumn{2}{|c|}{ TOTAL } & \multicolumn{2}{|c|}{ SI } & \multicolumn{2}{|c|}{ NO } & \multicolumn{2}{|c|}{ TOTAL } \\
\hline & $\mathbf{n}$ & $\%$ & $\mathbf{n}$ & $\%$ & $\mathbf{n}$ & $\%$ & $\mathbf{n}$ & $\%$ & $\mathbf{n}$ & $\%$ & $\mathbf{n}$ & $\%$ \\
\hline \multicolumn{13}{|l|}{ Soporte social } \\
\hline $\begin{array}{l}\text { Interés familiar por el Estado de } \\
\text { Salud }\end{array}$ & 24 & 60 & 16 & 40 & 40 & 100 & 61 & 76.3 & 19 & 23.8 & 80 & 100 \\
\hline $\begin{array}{l}\text { Calidad de Comunicación } \\
\text { Familiar }\end{array}$ & 23 & 57.5 & 17 & 42.5 & 40 & 100 & 61 & 76.3 & 19 & 23.8 & 80 & 100 \\
\hline \multicolumn{13}{|l|}{ Soporte económico } \\
\hline Disponibilidad Familiar & 10 & 25 & 30 & 75 & 40 & 100 & 44 & 55 & 36 & 45 & 80 & 100 \\
\hline \multicolumn{13}{|l|}{ Cuidado de interés familiar } \\
\hline Apoyo Económico Familiar & 23 & 57.5 & 17 & 42.5 & 40 & 100 & 33 & 41.3 & 47 & 58.8 & 80 & 100 \\
\hline $\begin{array}{l}\text { Cuidado por la Imagen y Aseo } \\
\text { Personal }\end{array}$ & 21 & 52.5 & 19 & 47.5 & 40 & 100 & 44 & 55 & 36 & 45 & 80 & 100 \\
\hline \multicolumn{13}{|l|}{ Cuidado de Asistencia social } \\
\hline $\begin{array}{l}\text { Participación Familiar en } \\
\text { Mantenimiento de la Salud }\end{array}$ & 17 & 42.5 & 23 & 57.5 & 40 & 100 & 39 & 48.8 & 41 & 51.3 & 80 & 100 \\
\hline Asistencia Médica de Control & 35 & 87.5 & 5 & 12.5 & 40 & 100 & 41 & 51.3 & 39 & 48.8 & 80 & 100 \\
\hline \multicolumn{13}{|l|}{ Cuidado de personal de apoyo } \\
\hline $\begin{array}{l}\text { Atención Personalizada } \\
\text { Permanente }\end{array}$ & 39 & 97.5 & 1 & 2.5 & 40 & 100 & 39 & 48.8 & 41 & 51.3 & 80 & 100 \\
\hline Percepción de Maltrato & 34 & 85 & 6 & 15 & 40 & 100 & 59 & 73.8 & 21 & 26.3 & 80 & 100 \\
\hline
\end{tabular}

\section{Resultados 2}

En la Tabla 4, en cuanto al grado de estudio, se observa que la mayoría de los encuestados que se perciben muy abandonados son los analfabetos en $44.4 \%$. La mayoría de los que se perciben abandonados tienen educación superior en $43.8 \%$. $Y$ los que se perciben ligeramente abandonados son los que tienen educación secundaria en $52.8 \%$.

En cuanto a la edad, se observa que la mayoría de los encuestados que se perciben muy abandonados tienen edades mayores de 91 en $50 \%$. La mayoría de los que se perciben abandonados están en un rango de 60 a 71 años en $28 \%$. Y la mayoría de los que se perciben ligeramente abandonados también entran dentro del rango 6071 en $40 \%$.
En cuanto al género, se observa que la mayoría de los encuestados que se perciben muy abandonados son de género masculino con $37,3 \%$. La mayoría de encuestados que se percibe $\mathrm{n}$ abandonados también son de género masculino con $37,3 \%$ y la mayoría de los que se sienten ligeramente abandonados constituyen el 50,8\% son del género femenino.

Con respecto al estado civil, se observa que dentro de los que se perciben muy abandonados son solteros con $38,6 \%$; dentro de los que solo se perciben abandonados, dentro de la mayoría de encuestados hay un empate entre los casados y divorciados con $28,6 \%$. La mayoría de los que se perciben ligeramente abandonados son divorciados con $42,9 \%$. 
Con respecto a la actividad económica, la mayoría de los encuestados que se perciben muy abandonados son jubilados con $41 \%$. Los que simplemente se perciben abandonados son en su mayoría empleados en 37,5\%. El mayor porcentaje dentro de los que de perciben como ligeramente abandonados son amas de casa en $100 \%$.

En cuanto a la religión, se observa que la mayoría de los encuestados que se perciben muy abandonados profesan la religión adventista en $50 \%$. La mayoría de los que se perciben abandonados profesan otras religiones distintas a las planteadas en $45,5 \%$. Y la mayoría de los que se perciben ligeramente abandonados son evangélicos en $54,5 \%$.

En cuanto a las personas con las que reside, se observa que la mayoría de los encuestados que se perciben muy abandonados viven solo con sus hijos en $43,2 \%$. La mayoría de encuestados que se percibe $n$ abandonados viven solos en $41,7 \%$ y la mayoría de los que se sienten ligeramente abandonados constituyen el $44,8 \%$ residen solo con su cónyuge.

Con respecto, al número de hijos, se observa que dentro de los que se perciben muy abandonados tienen más de 7 hijos en 42,9\%; dentro de los que solo se perciben abandonados la mayoría de encuestados no tienen hijos en $50 \%$. La mayoría de los que se perciben ligeramente abandonados tienen de 3 a 4 hijos en 47,2\%.

Con respecto a los hobbies, la mayoría de los encuestados que se perciben muy En la tabla 20 se observa que solo el género $(p=.012<\alpha)$ y la actividad socioeconómica $(p=.034<\alpha)$ guardan relación significativa con el abandono del adulto mayor. Mientras que en los demás casos como el grado de estudios $(p=.120>\alpha)$, la edad $(p=.917>\alpha)$, el estado civil $(p=.997>\alpha)$, la religión $(p=.492>\alpha)$, las personas con las que reside el encuestado $(p=.374>\alpha)$, el número de hijos $(p=.764>\alpha)$ y los hobbies $(p=.163>\alpha)$, no guardan relación significativa con el abandono del adulto mayor.

Tabla 4. Relación entre los factores sociodemográficos y el abandono del adulto mayor.

\begin{tabular}{lccc}
\hline \multicolumn{1}{c}{ Pearson } & Chi-cuadrado & gl & Sig. \\
\hline Grado de Estudio & 10.122 & 6 &, $120 \mathrm{a}$ \\
Edad & .950 & 4 &, $917 \mathrm{a}$ \\
Género & 8.876 & 2 &, $012^{\star}$ \\
Estado Civil & .539 & 6 &, $997 \mathrm{a}$ \\
Actividad Económica & 13.663 & 6 & $, 034 \mathrm{a},{ }^{*}$ \\
Religión & 5.412 & 6 &, $492 \mathrm{a}$ \\
Personas con las que & 8.635 & 8 &, $374 \mathrm{a}$ \\
Reside & 4.942 & 8 &, $764 \mathrm{a}$ \\
Número de Hijos & 11.752 & 8 &, $163 \mathrm{a}$ \\
Hobbies & & & \\
\hline
\end{tabular}




\section{Resultados 3}

En la Tabla 5 se observa que solo la edad $(p=.022<\alpha)$, guardan relación significativa con el abandono del adulto mayor. Mientras que en los demás casos como el género $(p=.181>\alpha)$ y la actividad socioeconómica $(p=.426>\alpha)$, el grado de estudios $(p=.152>\alpha)$, el estado civil $(p=.482>\alpha)$, la religión $(p=.441>\alpha)$, las personas con las que reside el encuestado $(p=.797>\alpha)$, el número de hijos $(p=.880>\alpha)$ y los hobbies $(p=.198>\alpha)$, no guardan relación significativa con el abandono del adulto mayor.

Tabla 5. Relación entre los factores sociodemográficos y el abandono del adulto mayor.

\begin{tabular}{lccc}
\hline \multicolumn{1}{c}{ Pruebas de chi-cuadrado de Pearson } & la percepción afectiva & Sig. \\
\hline Grado de Estudio & Chi-cuadrado & gl &, $152 a$ \\
Edad & 9,397 & 6 &, $022 a^{*}$ \\
Género & 11,475 & 4 &, 181 \\
Estado Civil & 3,424 & 2 &, $482 a$ \\
Actividad Económica & 5,496 & 6 &, $426 a$ \\
Religión & 5,976 & 6 &, $441 a$ \\
Personas con las que Reside & 5,844 & 6 &, $797 a$ \\
Número de Hijos & 4,625 & 8 &, $880 a$ \\
Hobbies & 3,742 & 8 & $, 198 a, c$ \\
\hline
\end{tabular}

\section{Resultados 4}

En la Tabla 6 se observa que ninguna variable sociodemográfica guarda relación significativa con el abandono del adulto mayor. Así tenemos: el grado de estudios $(p=.823>\alpha)$, la edad $(p=.928>\alpha)$, el género $(p=.225>\alpha)$ el estado civil $(p=.856>\alpha)$, la actividad socioeconómica $(\mathrm{p}=.368>\alpha)$, la religión $(p=.225>\alpha)$, las personas con las que reside el encuestado $(p=.133>\alpha)$, el número de hijos $(p=.621>\alpha)$ y los hobbies $(p=.247>\alpha)$, no guardan relación significativa con el abandono del adulto mayor.

Tabla 6. Relación entre los factores sociodemográficos y el abandono del adulto mayor.

\begin{tabular}{lccc}
\hline \multicolumn{1}{c}{ Pruebas de chi-cuadrado de Pearson } & la percepción de soporte & Sig. \\
\hline Grado de Estudio & Chi-cuadrado & gl &, $823 a$ \\
Edad & 2,890 & 6 &, $928 a$ \\
Género &, 875 & 4 &, 225 \\
Estado Civil & 2,984 & 2 &, $856 a$ \\
Actividad Económica & 2,608 & 6 &, $368 a$ \\
Religión & 6,514 & 6 &, $225 a$ \\
Personas con las que Reside & 8,190 & 6 &, $133 a$ \\
Número de Hijos & 12,426 & 8 &, $621 a$ \\
Hobbies & 6,234 & 8 &, $247 a$ \\
\hline
\end{tabular}




\section{Resultados 5}

En la Tabla 7, se observa que ninguna variable sociodemográfica guarda relación significativa con el abandono del adulto mayor. Así tenemos: el grado de estudios $(p=.645>\alpha)$, la edad $(p=.821>\alpha)$, el género $(p=.401>\alpha)$ el estado civil $(p=.383>\alpha)$, la actividad socioeconómica $(p=.632>\alpha)$, la religión $(p=.179>\alpha)$, las personas con las que reside el encuestado $(p=.322>\alpha)$, el número de hijos $(p=.324>\alpha)$ y los hobbies $(p=.496>\alpha)$, no guardan relación significativa con el abandono del adulto mayor.

Tabla 7. Relación entre los factores sociodemográficos y el abandono del adulto mayor.

\begin{tabular}{lccc}
\hline \multicolumn{1}{c}{ Pruebas de chi-cuadrado de Pearson } & la percepción de cuidado & Sig. \\
\hline Grado de Estudio & Chi-cuadrado & gl &, $645 a$ \\
Edad & 4,231 & 6 &, $821 a$ \\
Género & 1,532 & 4 &, 401 \\
Estado Civil & 1,829 & 2 &, $383 a$ \\
Actividad Económica & 6,366 & 6 &, $632 a$ \\
Religión & 4,332 & 6 &, $179 a$ \\
Personas con las que Reside & 8,904 & 6 &, $322 a$ \\
Número de Hijos & 9,247 & 8 &, $324 a$ \\
Hobbies & 9,217 & 8 & $, 496 a, c$ \\
\hline
\end{tabular}

\section{Resultados 5}

En la Tabla 8 se observa que las dimensiones, percepción de abandono afectivo y el percepción de abandono de soporte manifiestan un coeficiente de correlación $=, 472$ y el valor $\mathrm{p}=.000$. Indicando una correlación media directa y significativa.

Luego, se observa que las dimensiones, percepción de abandono afectivo y la percepción de abandono de cuidado personal manifiestan un coeficiente de correlación $=, 258$ y el valor $p=$ .002. Indicando una correlación baja directa y significativa.

Asimismo, se observa que las dimensiones, percepción de abandono de soporte y el percepción de abandono de cuidado personal manifiestan un coeficiente de correlación $=, 281$ y el valor $p=$ .001. Indicando una correlación baja directa y significativa. 
Tabla 8. Correlación entre los factores sociodemográficos y el abandono del adulto mayor.

\begin{tabular}{|c|c|c|c|c|}
\hline Tau_b & Kendall & $\begin{array}{c}\text { Percepción } \\
\text { de abandono }\end{array}$ & $\begin{array}{l}\text { Percepción de } \\
\text { abandono de }\end{array}$ & $\begin{array}{c}\text { Percepción } \\
\text { de abandono } \\
\text { de cuidado }\end{array}$ \\
\hline & Coeficiente de correlación & 1.000 &, $472^{\star \star}$ & $258^{\star \star}$ \\
\hline $\begin{array}{l}\text { Percepción de abandono } \\
\text { afectivo }\end{array}$ & Sig. (bilateral) & & .000 & .002 \\
\hline & $\mathrm{N}$ & 120 & 120 & 120 \\
\hline & Coeficiente de correlación &, $472^{\star \star}$ & 1.000 &, $281^{\star \star}$ \\
\hline $\begin{array}{l}\text { Percepción de abandono de } \\
\text { soporte }\end{array}$ & Sig. (bilateral) & .000 & & .001 \\
\hline & $\mathrm{N}$ & 120 & 120 & 120 \\
\hline & Coeficiente de correlación & $258^{\star \star}$ &, $281^{\star \star}$ & 1.000 \\
\hline $\begin{array}{l}\text { Percepción de abandono de } \\
\text { cuidado personal }\end{array}$ & Sig. (bilateral) & .002 & .001 & \\
\hline & $\mathrm{N}$ & 120 & 120 & 120 \\
\hline
\end{tabular}

\section{CONCLUSIONES}

El objetivo del presente estudio fue determinar la relación de los factores sociodemográficos con la percepción de abandono del adulto mayor, Lima, 2016. Hallándose que los factores sociodemográficos más de uno guardan relación significativa: género $(0,01)$ y actividad económica $(0,03)$. Es decir, el ser varón o mujer tienen diferente percepción con respecto al nivel de abandono; igualmente, el fecho de tener una dinámica económica (contar con recursos o no tenerlos) puede marcar una diferencia en la percepción de abandono que el adulto mayor pueda tener.

Por otro lado, los resultados evidencian que el grado de estudio, edad, estado civil, religión, personas con la que reside, número de hijos, hobbies no guardan relación con la percepción de abandono de los adultos. Es decir, el hecho que tenga menos estudio o más nivel de estudio igual percibiré el nivel de abandono. De la misma forma sea un adulto mayor más joven o adulto mayor más anciano igual sentirá nivel abandono. Igualmente, el hecho de ser casado (a) viudo (a) soltero (a) tampoco implicará que haya una diferencia en sentirme abandonado más que otro estado civil; todos sentiremos algún nivel de abandono.

El otro objetivo, fue determinar la relación de los factores sociodemográficos con la percepción afectiva en el abandono del adulto mayor, Lima, 2016. Hallándose que existe, relación significativa de al menos uno de los factores sociodemográficos con la percepción afectiva en el abandono del adulto mayor. Es decir, sólo la edad $(0,02)$ guardan relación significativa con la percepción afectiva de abandono. Dicho de otro modo, la edad; el hecho de ser un adulto mayor más joven o ser un adulto mayor de edad más avanzado puede estar implicando en grado de percepción que siente el adulto mayor propio a la edad; puedo esto estar asociado a la disminución de sus capacidades personales y enfermedades asociadas a la edad más madura un aspecto en el cual necesitarían estar más apoyado y no lo están.

Por otro lado, también los resultados describen que los factores sociodemográficos: grado de 
estudio, género, estado civil, actividad económica, religión, personas con la que reside, número de hijos y hobbies no guardan relación con la percepción afectiva de los adultos mayores. Dicho de otro modo, el hecho que tenga diferencia en el nivel educativo, sea hombre o mujer, sea nivel bajo o alto económicamente, indistintamente la religión que sea, y aún viva con poca o muchas personas o tenga pocos o muchos hijos y desarrolle cualesquiera de los hobbies igual va a estar expuesto a sentirse afectivamente abandonado.

En forma diferente, los resultados describen que los factores sociodemográficos: grado de estudio, edad, género, estado civil, actividad económica, religión, personas con la que reside, número de hijos y hobbies no guardan relación con la percepción de soporte de los adultos mayores. Dicho de otro modo, el hecho que tenga diferencia en el nivel educativo, sea adulto mayor más joven o más maduro, sea hombre o mujer, sea soltero (a) casado (a) viudo (a) divorciado (a), sea nivel bajo o alto económicamente, indistintamente la religión que sea, y aún viva con poca o muchas personas o tenga pocos o muchos hijos y desarrolle cualesquiera de los hobbies igualmente van a percibir el tener soporte o abandono por parte de sus familiares o seres queridos.

Igualmente, los resultados resaltan que los factores sociodemográficos: grado de estudio, edad, género, estado civil, actividad económica, religión, personas con la que reside, número de hijos y hobbies no guardan relación con la percepción de cuidado de los adultos mayores. Dicho de otro modo, el hecho que tenga diferencia en el nivel educativo, sea adulto mayor más joven o más maduro, sea hombre o mujer, sea soltero (a) casado (a) viudo (a) divorciado (a), sea nivel bajo 0 alto económicamente, indistintamente la religión que sea, y aún viva con poca o muchas personas o tenga pocos o muchos hijos y desarrolle cualesquiera de los hobbies igualmente van a percibir cuidado o abandono por parte de sus familiares o seres queridos.
Finalmente, producto de esta investigación se puede afirmar que con el sólo hecho de pasar a la etapa de adulto mayor; sin menoscabar la edad que tenga, o el nivel socioeconómico o el género o el estado civil que sea o la religión que práctica o con quién viva o la cantidad de hijos que tenga igual puede estar expuesto a sentirse abandonado; percibir afecto o abandono; o sentir que tiene soporte o no, o percibir tener cuidado por parte su familia o sentirse abandonado. Resultado que concretiza que el ser humano es integral en su bienestar que no sólo basta el aspecto emocional, sino el adulto mayor necesita estar cuidado y percibir que hay alguien que puede estar soportando todas sus necesidades. Resultados que descompaginan los planes de sectores de gobierno que sólo ven y se preocupan por el soporte y cuidado (económicamente hablando) sin embargo emocionalmente los adultos mayores en centros geriátricos se sienten abandonados; igualmente por otro lado los que viven en casas con sus familiares pueden tener soporte o cuidado y pueden sentirse emocionalmente abandonados 0 viceversa sentirse emocionalmente acompañados pero descuidados en el soporte de sus necesidades y cuidado.

\section{REFERENCIAS}

Bordignon, N. A. El desarrollo psicosocial de Eric Erikson . Lasallista Investig [Internet]. 2(2),5063. http://www.redalyc.org/pdf/695/69520210. pdf

Castillo, D. (2015). Enfermedades de los ancianos como causas de abandono familiar. http://dspace.unl.edu.ec/jspui/ bitstream/123456789/13457/1/tesis daniela castillo guam $\%$ c3\%81n.pdf

Cobo, D. J. (2010) Cambios físicos y psíquicos en la vejez. Atención socio sanitaria a Personas Domiciliadas [Internet]. 2010;30. http://www. logoss.net/uploads/cursos/temamuestra/ tema271.pdf 
Gurevicz, M. y Toro, C. (2010). La Teoría De La Gestalt. 1804;1-8. Available from: file://C:/ Users/alumno.l4/Downloads/Teor\%C3\%ADa_ de_la_Gestalt.pdf

INEI (2016). Población Adulta Mayor. En línea https://www.inei.gob.pe/biblioteca-virtual/ boletines/ninez-y-adulto-mayor/1/

Instituto de Mayores y Servicios Sociales (IMSERSO, 2012). Las personas mayores en españa. datos estadísticos estatales y por comunidades autonomas. ), Informe 2012. Catálogo de publicaciones de la Administración General del Estado. 2014. 286 p
Merchán, E., Cifuentes, R. (2014) . Teorías Psicosociales del Envejecimiento. J Chem Inf Model [Internet]. 2013;53(9):1689-99. Available from: http://asociacionciceron.org/wpcontent/uploads/2014/03/00000117-teoriaspsicosociales-del-envejecimiento.pdf

Organización de las Naciones Unidas (ONU, 2007). El desarrollo en un mundo que envejece. 2007; Estudio Económico y Social Mundial 2007

Organización Mundial de la Salud (OMS, 2015). Informe mundial sobre el envejecimiento y la salud. J Chem Inf Model. 2015;53(9),1689-99 